\title{
Spatiotemporal patterns of smooth muscle cell changes in ascending aortic dilatation with bicuspid and tricuspid aortic valve stenosis: Focus on cell-matrix signaling
}

Alessandro Della Corte, MD, ${ }^{a}, *$ Cesare Quarto, MD, ${ }^{\mathrm{a}, *}$ Ciro Bancone, MD, ${ }^{a}$ Clotilde Castaldo, MD, ${ }^{\mathrm{b}}$

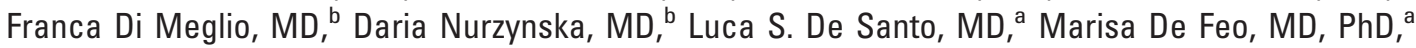
Michelangelo Scardone, MD, ${ }^{\text {a }}$ Stefania Montagnani, MD, and Maurizio Cotrufo, MD ${ }^{\mathrm{a}}$

Supplemental material is available online.
From the Department of Cardiothoracic and Respiratory Sciences, Second University of Naples, V. Monaldi Hospital, Naples, It$\mathrm{aly}^{\mathrm{a}}$; and the Department of Biomorphological and Functional Sciences, "Federico II" University, Secondo Policlinico, Naples, Italy. ${ }^{\mathrm{b}}$

This study was supported by a grant (P.R.I.N. co-funding 2006; prot. 2006063487) from the Italian Ministry for University and Research.

Read at the Eighty-seventh Annual Meeting of The American Association for Thoracic Surgery, Washington, DC, May 5-9, 2007.

Received for publication May 1, 2007; revisions received Aug 23, 2007; accepted for publication Sept 20, 2007.

Address for reprints: Alessandro Della Corte, MD, Via P. Neruda, 6, 81031 Aversa CE, Italy (E-mail: aledellacorte@libero.it).

$* \mathrm{PhD}$ program, "Medical and Surgical Physiopathology of the Cardio-Respiratory System and Associated Biotechnologies," Second University of Naples, Italy.

J Thorac Cardiovasc Surg 2008;135:8-18

$0022-5223 / \$ 34.00$

Copyright $(\odot) 2008$ by The American Association for Thoracic Surgery

doi:10.1016/j.jtcvs.2007.09.009
Objective: The present study examined temporal and spatial patterns of extracellular matrix and smooth muscle cell changes in the ascending aorta with bicuspid and tricuspid aortic valve stenosis.

Methods: Wall specimens were retrieved from both the greater and the lesser curvature ("convexity" and "concavity") of 14 nonaneurysmal and 12 aneurysmal aortas (aortic ratios 1.2 and 1.5, respectively) and from 3 heart donors (normal). Immunochemistry was performed for detection of apoptotic (terminal deoxynucleotidyl transferase-mediated dUTP nick end labelling [TUNEL]-positive) and proliferating (Ki-67-positive) smooth muscle cells and for semiquantification of matrix proteins (collagens, fibronectin, tenascin, laminin). Co-immunoprecipitation assessed the extent of Bcl-2-modifying factor binding to Bcl-2, indicating a matrixderived cytoskeleton-mediated proapoptotic signaling. Polymerase chain reaction allowed for quantification of messenger RNA expression for Bcl-2.

Results: In both bicuspid and tricuspid aneurysms, fibrillar collagens were reduced, whereas fibronectin and tenascin were increased compared with those in normal conditions. These matrix alterations were already evident in bicuspid nonaneurysmal aortas at the convexity, with significant elevation of apoptotic indexes $(P=.02$ bicuspid vs normal; $P=.48$ tricuspid vs normal). Apoptotic indexes correlated with aortic dimensions only in tricuspid aortas $(P=.01)$. No significant increase in Ki-67 was found. Higher levels of Bcl-2-modifying factor-Bcl-2 binding were found in bicuspid nonaneurysmal aorta versus tricuspid $(P=.03)$ and normal aortas $(P=$ $.01)$. Bcl-2 messenger RNA expression was reduced in the bicuspid aorta versus normal $(P=.08)$.

Conclusions: Smooth muscle cell apoptosis with bicuspid aortic valve stenosis occurred before overt aortic dilation, mainly at the convexity, where wall stress is expectedly higher. In this setting, a matrix-dependent proapoptotic signaling was evidenced by increased Bcl-2-modifying factor-Bcl-2 binding. Stress-dependent bicuspid aortic valve matrix changes may trigger early apoptosis by inducing cytoskeletal rearrangement.

$\mathrm{T}$ he traditional definition of medial degeneration of the ascending aorta includes the association of smooth muscle cell (SMC) loss, owing to apoptosis, ${ }^{1,2}$ and extracellular matrix (ECM) rearrangement. ${ }^{3,4}$ Recently, remarkable improvements have been achieved in the knowledge of the mechanisms underlying ascending aortic dilations, ${ }^{1-4}$ but without identifying definite pathogenetic sequences. Indeed, medial degeneration can underlie a variety of anatomoclinical conditions (eg, the senile aorta, poststenotic dilations, idiopathic or syndromic aneurysms, dissections), suggesting different possible mechanisms of lesion 


$$
\begin{aligned}
& \text { Abbreviations and Acronyms } \\
& \begin{aligned}
\text { AI } & =\text { apoptotic index } \\
\text { BAV } & =\text { bicuspid aortic valve } \\
\text { Bmf } & =\text { Bcl-2-modifying factor } \\
\text { DAPI } & =4^{\prime}, 6 \text {-diamidino- } 2^{\prime} \text {-phenylindole } \\
\text { ECM } & =\text { extracellular matrix } \\
\text { GAPDH } & =\text { glyceraldehyde-3-phosphate dehydrogenase } \\
\text { MMP } & =\text { matrix metalloproteinases } \\
\text { mRNA } & =\text { messenger RNA } \\
\text { PBS } & =\text { phosphate-buffered saline } \\
\text { PCR } & =\text { polymerase chain reaction } \\
\text { SMC } & =\text { smooth muscle cell } \\
\text { TAV } & =\text { tricuspid aortic valve } \\
\text { TUNEL } & =\text { terminal deoxynucleotidyl transferase- } \\
& \text { mediated dUTP nick end labeling }
\end{aligned}
\end{aligned}
$$

development. ${ }^{1}$ In particular, aortic dilations associated with congenital bicuspid aortic valve (BAV) are known to differ from those with tricuspid aortic valve (TAV) disease in terms of histopathology, ${ }^{2}$ anatomic configuration, ${ }^{5}$ and natural history. ${ }^{6}$

In our preliminary investigation, we $\mathrm{e}^{4}$ defined medial ECM changes in BAV-associated dilations, observing a decrease in collagen content versus normal aorta and an increase in fibronectin and the expression of tenascin. However, no comparison with TAV-associated dilations and no distinction between early changes and mature lesions were performed. The present study was undertaken as a more in-depth continuation of the previous one, ${ }^{4}$ with the aim to assess spatial and temporal patterns of ECM protein and SMC changes in BAV- and TAV-associated aortic dilations. Moreover, inasmuch as changes in the ECM composition, including those previously observed in BAV aortopathy, ${ }^{3,4}$ are known to possibly influence SMC phenotype, proliferation, survival, and synthetic activity, ${ }^{7,8}$ we focused on the hypothesis that SMC apoptosis in ascending dilations could be provoked by matrix-derived signaling. Anoikis (the ancient Greek word for homelessness) is the term indicating apoptosis caused by the loss of normal adhesion of cells to a normally organized ECM, occurring via altered cell "tensegrity," the tensional integrity of the cytoskeleton. ${ }^{9}$ Matrix disarray and/or cell detachment can induce loss of cytoskeletal integrity, cell shape changes, and eventually cell death ${ }^{9,10}$ : thus, "amorphosis" is considered as a typical feature/modality of anoikis. ${ }^{10,11}$ Proapoptotic cues are known to be transmitted to cells by the ECM in both the settings of vascular physiology and disease. ${ }^{9}$ In the present study, we hypothesized that matrix-derived proapoptotic signals could be involved in the development of BAV aortopathy.

\section{Patients and Methods \\ Study Patients}

The study included 26 patients with aortic valve stenosis (mean age $59 \pm 15$ years, $65 \%$ male) undergoing aortic valve replacement and/or ascending aorta replacement. Valve morphology (BAV/TAV) was defined on the basis of concordant echocardiographic diagnosis, surgical inspection, and pathologic examination. In case of discordances, patients were considered noneligible. Twelve patients had degenerative stenosis of a TAV, and 14 had a stenotic congenital BAV. Respective TAV and BAV subgroups were comparable for clinical and echocardiographic data (Table E1). No patient had inherited connective tissue abnormalities, atherosclerotic aneurysm, or associated aortic regurgitation (more than mild). To define temporal patterns of medial change progression, we distinguished subgroups according to aortic dimensions: nonaneurysmal aortas (mild dilations) were defined as an aortic ratio (between echocardiographically measured maximal diameter and expected diameter, based on age and body surface area) less than 1.4 (corresponding, on average, to a diameter of $4.5 \mathrm{~cm}$ ), and aneurysms were defined as a ratio greater than 1.4.

\section{Sample Retrieval Protocol}

At surgical inspection, all BAV-associated aortic aneurysms appeared asymmetric, with predominant bulging of the right anterolateral side of the vessel. This configuration was found also in 3 TAV aneurysms (60\%), whereas the other 2 showed a fusiform, symmetric dilation at both the outer (convexity) and inner curves (concavity). The pattern of cusp fusion was right-left coronary in 12 BAV patients and right-noncoronary in the other 2. To define spatial patterns of lesion expression, according to a previously introduced protocol, ${ }^{4}$ soon after the aortotomy was performed, we retrieved 2 full-thickness aortic wall samples distal to the sinotubular junction (maximal dilation level) in each patient: one at the convexity and the other at the concavity (from the two ends of the transverse aortotomy in patients without indication to concomitant ascending aorta replacement). Wall specimens were retrieved at the same levels also from 3 normotensive heart donors during multiorgan procurement ( 2 men, 1 woman; mean age $40 \pm 3$ years; mean diameter $3.4 \pm 0.15 \mathrm{~cm}$ ). Thus, 58 specimens overall were available for examination. The study was approved by our institution's ethical committee and patients gave informed consent.

\section{Immunohistochemistry of Matrix Proteins}

Specimens $(n=52)$ were fixed in buffered $10 \%$ formalin, embedded in paraffin, and sectioned. Serial $4 \mu \mathrm{m}$-thick sections of aortic specimens were deparaffinized, covered with primary monoclonal antibodies against type I collagen, tenascin- $\mathrm{C}$, and laminin or polyclonal antibody for fibronectin (Sigma-Aldrich, St Louis, Mo), and type III collagen (Santa Cruz Biotechnology Inc., Santa Cruz, Calif), and incubated in a moist chamber for 1 hour at $37^{\circ} \mathrm{C}$. After washings in phosphate-buffered saline (PBS), they were covered with fluoresceinated secondary antibodies (Sigma-Aldrich) and reincubated. Nuclei were stained with propidium iodide, and then sections were mounted in Vectashield mounting medium (Vector Laboratories, Burlingame, Calif) and observed with Leica DMLB fluorescence microscope (Leica Microsystems, Inc, Bannockburn, Ill). Moreover, a Zeiss LSM-510 confocal microscope (Karl Zeiss 
A

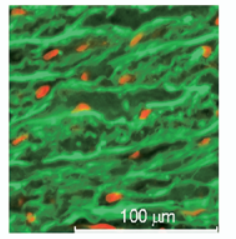

normal concavity

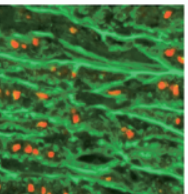

TAV/dil conc

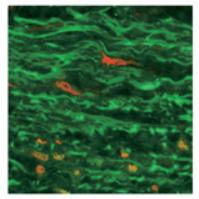

TAV/aneu conc

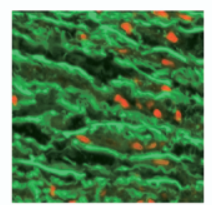

TAV/dil conv

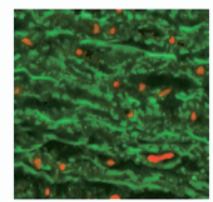

TAV/aneu conv
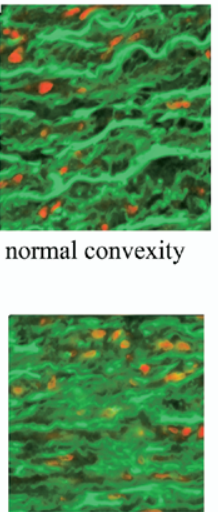

$\mathrm{BAV} /$ dil conc

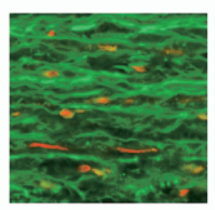

BAV/aneu conc
B

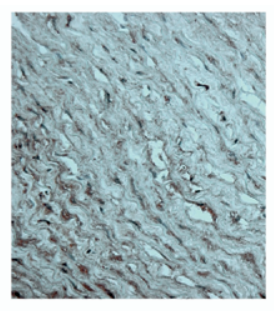

normal concavity

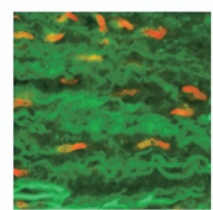

BAV/dil conv

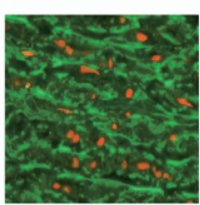

BAV/aneu conv

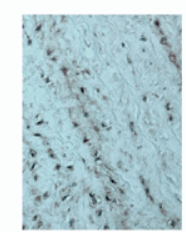

TAV/dil conv

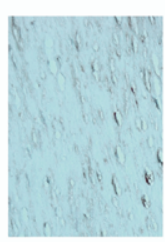

TAV/aneu conv

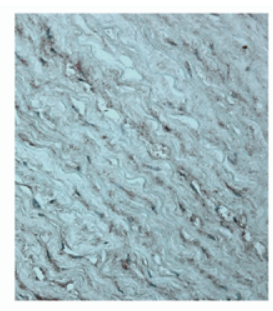

normal convexity

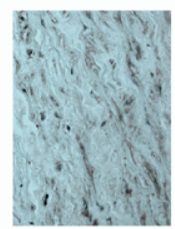

BAV/dil conc

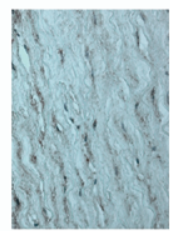

$\mathrm{BAV} / \mathrm{aneu}$ conc

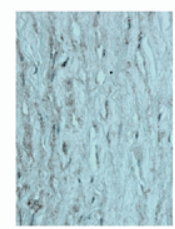

BAV/dil conv

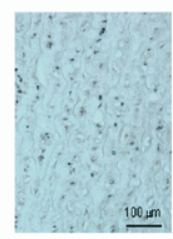

BAV/aneu conv

Figure 1. Immunostaining for laminin is shown as a representative example of the observed patterns of ECM changes. A, Immunofluorescence method, confocal miscroscopy. Green fluorescence = laminin; red fluorescence = propidium iodide (nuclei). B, Immunoperoxidase method, light microscopy, 200x; laminin stained brown). Both methods indicated that in BAV patients laminin decrease was earlier (ie, already evident in small-size dilations) and more asymmetrical (ie, more severe at the convexity than at the concavity) than in TAV patients. All images were from the inner portion of the media. ECM, Extracellular matrix; BAV, bicuspid aortic valve; TAV, tricuspid aortic valve.

International, Jena, Germany) was used to obtain full-thickness reconstructions of the aortic wall to describe spatial distribution of proteins. To obviate the possible interference of elastin autofluorescence, however, quenched by pretreatment with toluidine blue $0.1 \%$ solution, we also performed the immunoperoxidase method for countercheck, using the standard avidin-biotin-peroxidase complex technique and the L.V. Dako LSAB kit (DAKO A/S, Carpinteria, Calif) after antigen retrieval by pressure cooking. After quenching in 3\% hydrogen peroxide and blocking, the sections were incubated with primary antibodies for the aforementioned ECM proteins overnight at $4{ }^{\circ} \mathrm{C}$. Biotinylated secondary antibody and streptavidin conjugated to horseradish peroxidase were subsequently applied with color development with 3,3'diaminobenzidine and hematoxylin counterstaining. Negative control slides in the absence of primary antibody were included for each staining. Samples were evaluated with both methods by 3 independent observers blinded to the register that matched specimens with patient clinical data, using a grading scale for semiquantification of staining, from none (0) to intense (4). Owing to the overall good correspondence between fluorescence and peroxidase assessments, each specimen was assigned 1 score (averaging the 6 scores from the 2 methods).

\section{Assessment of SMC Density, Apoptosis, and Proliferation}

Wall fragments from the concavity and convexity of the ascending aorta ( $\mathrm{n}=54)$ were washed in PBS, fixed in $10 \%$ buffered formalin, paraffin-embedded, and sliced in 4- $\mu \mathrm{m}$ serial sections. After deparaffinization, the ApopTag Plus Fluorescein In Situ Apoptosis Detection Kit (Chemicon, Temecula, Calif) was used, according to the manufacturer protocol. In brief, sections were pretreated with proteinase $\mathrm{K}$ for 15 minutes. Pre-equilibrated sections were covered with terminal deoxynucleotidyl transferase working solution and incubated for 1 hour at $37^{\circ} \mathrm{C}$ in a humidified chamber. The reaction was stopped by incubation with stop/wash buffer. After three washes with PBS, antidigoxigenin fluorescein isothiocyanate conjugate (Sigma-Aldrich) was applied to the slides for 30 minutes at room temperature. For characterization of apoptotic cells, sections were subsequently incubated with antismooth muscle $\alpha$-actin antibody (Sigma-Aldrich) for 1 hour at $37^{\circ} \mathrm{C}$ in a humidified chamber. After washes in PBS, rhodamineconjugated secondary immunoglobulin $\mathrm{G}$ antibody (Jackson ImmunoResearch Laboratories, Inc, West Grove, Pa) was applied to the sections for 1 hour at $37^{\circ} \mathrm{C}$. Cell nuclei were counterstained 

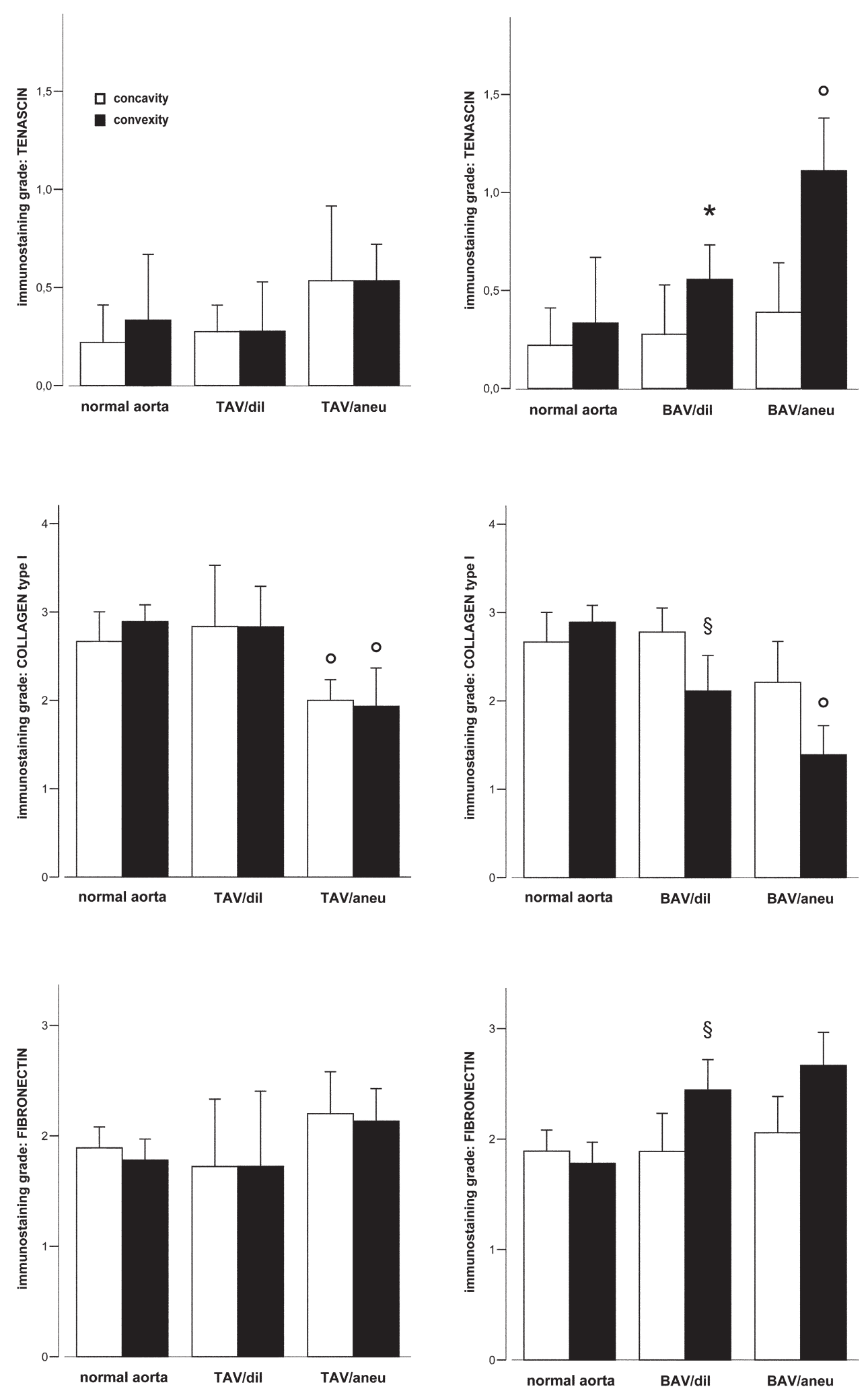

Figure 2. Patterns of ECM changes by semiquantitative assessment of collagen type $I$, fibronectin, and tenascin immunostainings. ${ }^{*} \boldsymbol{P}=.05 \mathrm{BAV}$ versus TAV dilation; $\S \boldsymbol{P}<.05 \mathrm{BAV}$ versus normal $(\boldsymbol{P}=.016$ for collagen type $\mathrm{I} ; \boldsymbol{P}$ $=.008$ for fibronectin) and versus TAV dilation $\left(\boldsymbol{P}=.008\right.$ for collagen type $\mathrm{I} ; \boldsymbol{P}=.037$ for fibronectin); ${ }^{\circ} \boldsymbol{P}<.05$ aneurysm versus respective dilation $(P=.007$ for collagen type $I$ in $B A V ; P=.03$ and $P=.01$ for collagen type I in TAV; $P=.002$ for tenascin in BAV); $P<.01$ for all differences between convexity and concavity in BAV. $P<$ .05 (not significant) for all other comparisons. ECM, Extracellular matrix; TAV, tricuspid aortic valve; BAV, bicuspid aortic valve. 
with 4',6-diamidino-2'-phenylindole (DAPI), and the sections were washed in PBS and mounted under a glass coverslip. The slides were examined with a fluorescence microscope (Leica Microsystems) by 3 independent observers blinded to the register matching specimens with patient clinical data: the number of SMCs in $1 \mathrm{~mm}^{2}$ (SMC density) and the rate of apoptotic SMCs (apoptotic index, AI) were determined counting 1000 nuclei in each section. For investigation of SMC proliferation, double immunostaining was performed $(n=24)$ with the aforementioned anti-actin antibody (Sigma-Aldrich) and primary rabbit polyclonal antibodies against the Ki-67 antigen (NovoCastra Laboratories Ltd, Newcastle upon Tyne, United Kingdom), previously unmasked by pressure cooking in 10-mmol citric acid, $\mathrm{pH}$ 6. Ki-67 is expressed during late $\mathrm{G} 1, \mathrm{~S}, \mathrm{G} 2$, and $\mathrm{M}$ phases of the cell cycle, not in early G1 and G0. ${ }^{12}$

\section{Bcl-2-modifying Factor-Bcl-2 Coimmunoprecipitation and Bcl-2 Messenger RNA Quantification}

To assess the presence and degree of matrix-dependent cytoskeleton-mediated proapoptotic signals in aortic dilations, we quantified the interaction of Bcl-2-modifying factor (Bmf) with Bcl-2. $\mathrm{Bmf}$ is a proapoptotic $\mathrm{BH}-3$ only protein normally sequestered to the myosin $\mathrm{V}$ actin motor complex ${ }^{13}$ : when released by actin cytoskeletal reorganization after altered matrix rigidity ${ }^{9-11,14}$ or cell-matrix detachment, ${ }^{9,11}$ it binds to Bcl-2, initiating apoptosis. The tissue fragments were fast frozen in liquid nitrogen and pulverized. The samples $(n=42)$ were suspended in a lysis buffer containing $50 \mathrm{mmol} / \mathrm{L}$ Tris- $\mathrm{HCl}(\mathrm{pH} \mathrm{7.4)}, 5 \mathrm{mmol} / \mathrm{L}$ ethylenediaminetetraacetic acid, $250 \mathrm{mmol} / \mathrm{L} \mathrm{NaCl}, 0.1 \%$ Triton X-100 supplemented with proteases inhibitors $(1 \mathrm{mmol} / \mathrm{L}$ dithiothreitol, 2 $\mathrm{mmol} / \mathrm{L}$ phenylmethylsulfonyl fluoride, $2 \mu \mathrm{g} / \mathrm{mL}$ aprotinin, and 10 $\mu \mathrm{g} / \mathrm{mL}$ leupeptin), incubated on ice for 30 minutes to obtain total cell lysate, and centrifuged at $14000 \mathrm{~g}$ for 20 minutes. The protein concentration in the supernatant was determined with the BioRad Protein Assay (Bio-Rad Laboratories, Hercules, Calif). The lysates containing $300 \mu \mathrm{g}$ of proteins were incubated with anti-Bcl-2 antibody (Santa Cruz Biotechnology) at $+4^{\circ} \mathrm{C}$ overnight, followed by incubation with protein $\mathrm{G}$ agarose (Invitrogen Corporation, Carlsbad, Calif) for 3 hours. Immunoprecipitates were washed 3 times and collected beads were boiled in Laemmli loading buffer (Bio-Rad Laboratories). The presence of Bmf bound to Bcl-2 was analyzed by electrophoresis on $12 \%$ sodium dodecylsulfate-poliacrylamide gel and Western blot using anti-Bmf antibody (Santa Cruz Biotechnology). ${ }^{13}$ An incubation of tissue lysates with nonspecific immunoglobulin $\mathrm{G}$ at the same concentration was used as a negative control for immunoprecipitation, and the absence of signal in control samples confirmed specificity. An internal control sample was run on each gel for normalization. For corollary assessment of Bcl-2 expression, total RNA was isolated by lysis ( $\mathrm{n}$ $=20 ; 3$ normal aortas and $7 \mathrm{BAV}$-associated mild dilations) in TRIzol solution (GIBCO BRL, Invitrogen Life Technologies, Rockville, Md). After the yield and integrity control of each RNA sample, 2.5 to $5 \mu \mathrm{g}$ of total RNA was reverse-transcribed by using the First-Strand cDNA Synthesis Kit (Amersham Pharmacia Biotech, Arlington Heights, Ill) with the random hexamer primers. The same volume $(2 \mu \mathrm{L})$ of products from each sample was used for subsequent polymerase chain reaction (PCR) amplification with the primers reported in Appendix E1. Samples without com- plementary DNA served as negative controls for PCR amplifications. See Appendix E1 for the PCR program. The amplified products were analyzed by electrophoresis in $2 \%$ agarose gel containing ethidium bromide, and photographs were taken under ultraviolet illumination. Bcl-2 messenger RNA (mRNA) expression was quantified by densitometry scanning and normalized against glyceraldehyde-3-phosphate dehydrogenase (GAPDH) controls.

\section{Statistical Analysis}

Paired $t$ test (convexity vs concavity) and the Student $t$ test (BAV vs TAV) were used for comparisons. One-way analysis of variance with Bonferroni post hoc correction was used for comparisons between normal, dilated, and aneurysmal aortas. Correlations were analyzed by computing the Pearson coefficient.

\section{Results}

\section{ECM Protein Expression}

Immunohistochemistry with both fluorescence and peroxidase methods allowed for semiquantitative definition of changes in the expression of ECM proteins and in their localization within the medial layer. In aneurysmal aortas, both with BAV and TAV, media thickness was constantly decreased $(1.17 \pm 0.2 \mathrm{~mm})$ compared with normal (1.54 \pm $0.05 \mathrm{~mm} ; P<.001)$. Collagen type I, collagen type III, and laminin were reduced compared with normal, whereas fibronectin was increased; tenascin was scarcely detected in normal aortas, whereas it was present in aneurysms. The inner media (Figure 1) showed the most marked reduction in laminin and collagen type I content, which gradually increased toward the adventitia. In BAV aneurysms, all the aforementioned changes were more severe at the convexity than at the concavity, whereas TAV aneurysms showed symmetrical changes (Figure 2). Also, in BAV small-size dilations, the convexity showed reduction of collagens and laminin and increase of fibronectin and tenascin (although less severe compared with aneurysms), whereas the concavity showed near-normal amounts of proteins (Figure 2). In TAV mildly dilated aortas, ECM protein expression was more heterogeneous: normal mean amounts of proteins were observed in all TAV dilations, except for fibronectin levels in 4 cases (slightly increased in 2, decreased in 2) and collagen I in 3 (increased). The lamellar structures evidenced in normal aorta appeared thinned, fragmented, and disarrayed in patients, especially at BAV convexity.

\section{SMC Apoptosis Density and Proliferation}

In the overall group of diseased aortas, the rates of terminal deoxynucleotidyl transferase-mediated dUTP nick end labeling (TUNEL)-positive SMC nuclei were increased compared with normal $(P<.001)$. In TAV dilations, the AIs were remarkably heterogeneous, likely accounting for the lack of statistically significant difference versus normal aortas. In particular, of 14 available TAV dilation specimens, 8 showed normal AIs and 6 had AIs even higher than 

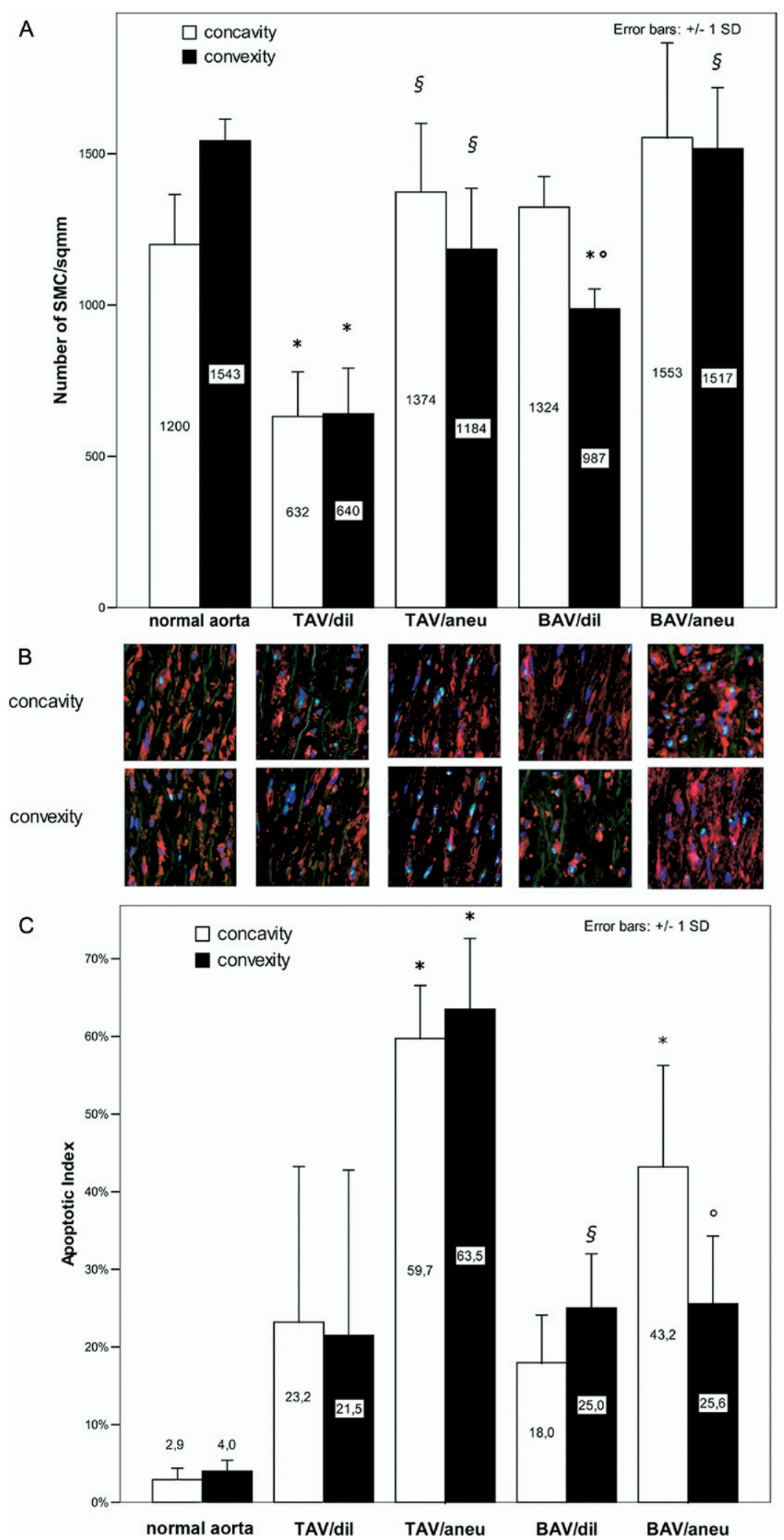

Figure 3. SMC changes in TAV and BAV poststenotic dilation. A, Comparison of SMC density between subgroups. Smooth muscle cellularity was generally higher in BAV than in TAV dilations. The temporal pattern of SMC density changes found in TAV was observed also at BAV convexity. ${ }^{*} \boldsymbol{P}<.05$ versus normal; $\boldsymbol{\S P}<.05$ versus respective dilation and $P=$ not significant versus normal; ${ }^{\circ} P<.05$ versus respective concavity. B, Representative images illustrating SMC density and apoptosis in the study subgroups (red fluorescence $=\alpha$-smooth muscle actin positivity; blue fluorescence $=$ DAPI staining for nuclei; cyan-green fluorescence $=$ TUNEL positivity). C, Comparative graph of apoptotic indexes (AI) in the subgroups. BAV aortas showed a TAV-like pattern of Al increase with increasing aortic dimensions only at the concavity. At the convexity, the BAV aorta showed a significant initial Al increase compared with normal, without further significant increase in aneurysms compared to dilations. ${ }^{*} \boldsymbol{P}<.05$ versus normal and versus respective dilation; $\$ P<.05$ versus normal; ${ }^{\circ} \boldsymbol{P}<.05$ versus respective concavity (paired $t$ test). SMC, Smooth muscle cell; TAV, tricuspid aortic valve; $B A V$, bicuspid aortic valve; $S D$, standard deviation. 
those found in BAV dilations. In BAV dilations, AIs were significantly higher than normal $(P=.02)$ only at the convexity (Figure 3 ). In aneurysms, the AIs increased significantly compared with small-size dilations at both concavity $(P=.024)$ and convexity $(P=.017)$ in TAV patients, whereas in BAV aneurysms they increased only at the concavity $(P=.02)$ with significant difference versus the convexity $(P=.02)$. There was a significant correlation between aortic size and AI in TAV patients (concavity: $r=$ $0.69 ; P=.038$; convexity: $r=0.72 ; P=.030$ ), but not in BAV patients (concavity: $r=0.50 ; P=.17$; convexity: $r=$ $0.03 ; P=.92)$. Normal aortas presented nonsignificantly greater SMC density at the convexity $(1543 \pm 69$ cells/ $\left.\mathrm{mm}^{2}\right)$ than at the concavity $\left(1200 \pm 166\right.$ cells $/ \mathrm{mm}^{2} ; P=$ .12). Small-size dilations showed decreased SMC density compared with normal aorta, whereas aneurysms showed an increase compared with dilations, with near-normal values $(P>.05$ in aneurysm subgroups versus normal; Figure 3). TAV patients showed this 2-phase temporal pattern of SMC density changes both at the concavity and at the convexity, BAV patients only at the convexity: in BAV concavity, a steady trend in SMC density was observed (dilations versus normal: $P=.84$; aneurysms versus dilations: $P=.77$ ). Few scattered smooth muscle actin-positive cells co-stained with Ki-67 $(0.06 \% \pm 0.03 \%)$ in the media of normal aortas. In aneurysms (5 BAV, $4 \mathrm{TAV}$ ), the mean rate of medial $\mathrm{Ki}-67$-positive $/ \alpha$-actin-positive cells was $0.07 \% \pm 0.05 \%$ $(P=.32$ versus normal; Figure E1). Owing to limited tissue availability for experiments, no comparison between subgroups could be performed.

\section{Proapoptotic Versus Prosurvival Signaling}

Low levels of Bmf-Bcl-2 binding were present in normal aortas, showing that it may be a constitutive phenomenon; however, it was increased in patients, considered as one group, compared with normal (concavity: $0.92 \pm 0.17$ vs $0.72 \pm 0.01 ; P=.07$; convexity: $1.10 \pm 0.24$ vs $0.73 \pm$ $0.01 ; P=.02)$. Grouped together, all BAV patients presented higher levels of Bmf-Bcl-2 binding than the TAV group at the convexity $(1.21 \pm 0.24$ vs $0.96 \pm 0.16 ; P=$ .03 ; concavity: $0.93 \pm 0.19$ vs $0.90 \pm 0.16 ; P=.83$ ). As to the temporal patterns of Bmf-Bcl-2 binding, it increased in mild dilations compared with normal, but no further increase was detectable in the aneurysm phase (Figure 4). Bmf-Bcl-2 level did not correlate statistically with the AI. The expression of Bcl-2 mRNA was lower in patients than in normal subjects, although statistical significance was not reached (optical density ratio to GAPDH: $0.20 \pm 0.09$ vs $0.29 \pm 0.2 ; P=.08)$.

\section{Discussion}

The main findings of this study may be summarized as follows: (1) BAV and TAV stenosis showed different tem- poral patterns of ascending aorta medial remodeling, in that BAV was associated with significant SMC apoptosis even before overt enlargement of the vessel, whereas TAV was characterized by high variability in SMC apoptosis rates at this early stage; (2) similarly, ECM remodeling was earlier with BAV, being characterized by collagen and laminin reduction, fibronectin increase, and tenascin expression, whereas only a proportion of TAV patients showed early ECM changes, in part discordant in nature with those seen with BAV; (3) BAV and TAV stenosis also showed different spatial patterns of medial remodeling: asymmetric in BAV aortopathy, with the convexity more severely involved than the concavity, and symmetric in TAV; (4) although present also in normal aorta, Bmf-Bcl-2 binding was significantly increased, in BAV small-size dilations, at the convexity, with concomitant reduction of Bcl-2 expression, indicating a net proapoptotic alteration of cell-matrix signaling. These represented novel insights into the physiopathology of ascending aorta dilations in the presence of BAV stenosis.

\section{Early and Late SMC Changes}

To our knowledge, only one previous report ${ }^{2}$ showed early apoptosis in the nonaneurysmal BAV aorta, although it included patients operated on for aortic stenosis, regurgitation, coarctation, and coronary artery disease in one group. However, in studies assessing aortic wall changes, patient samples should be homogeneous for valve function, inasmuch as important differences have been found between BAV stenosis and regurgitation in terms of epidemiology and morphologic/molecular features of ascending aortic dilation ${ }^{4}$ : valve function can influence even the natural history of dilation. ${ }^{6}$ Other investigations on SMC apoptosis in BAV aortopathy analyzed only end-stage aneurysmal tissues. ${ }^{1,2}$

Our findings indicate that the cellular and extracellular processes underlying aortic enlargement are already operative in the normally sized or slightly dilated BAV aorta (mean diameter $3.8 \mathrm{~cm}$ ), although limited to the convexity. The evidence of a "preclinical" aortopathy may add to the current debates on whether and how to concomitantly address the ascending aorta at the time of surgery for BAV stenosis. ${ }^{15}$ The recent studies on matrix metalloproteinases ${ }^{3}$ (MMPs), as well as gene mutational analyses,${ }^{16}$ may potentially pave the way for development of new diagnostic and predictive strategies that may help to cope with this clinical challenge.

With increasing aortic dimensions (mean diameter $4.9 \mathrm{~cm}$ ), ECM rearrangement at BAV convexity continued, whereas SMC density increased back to normal levels. A similar temporal pattern was observed in the TAV setting, although accompanied by a significant increment of AIs, in apparent contrast with the increase in cellularity. Also, Tang and coworkers ${ }^{17}$ have recently found comparable SMC density in 


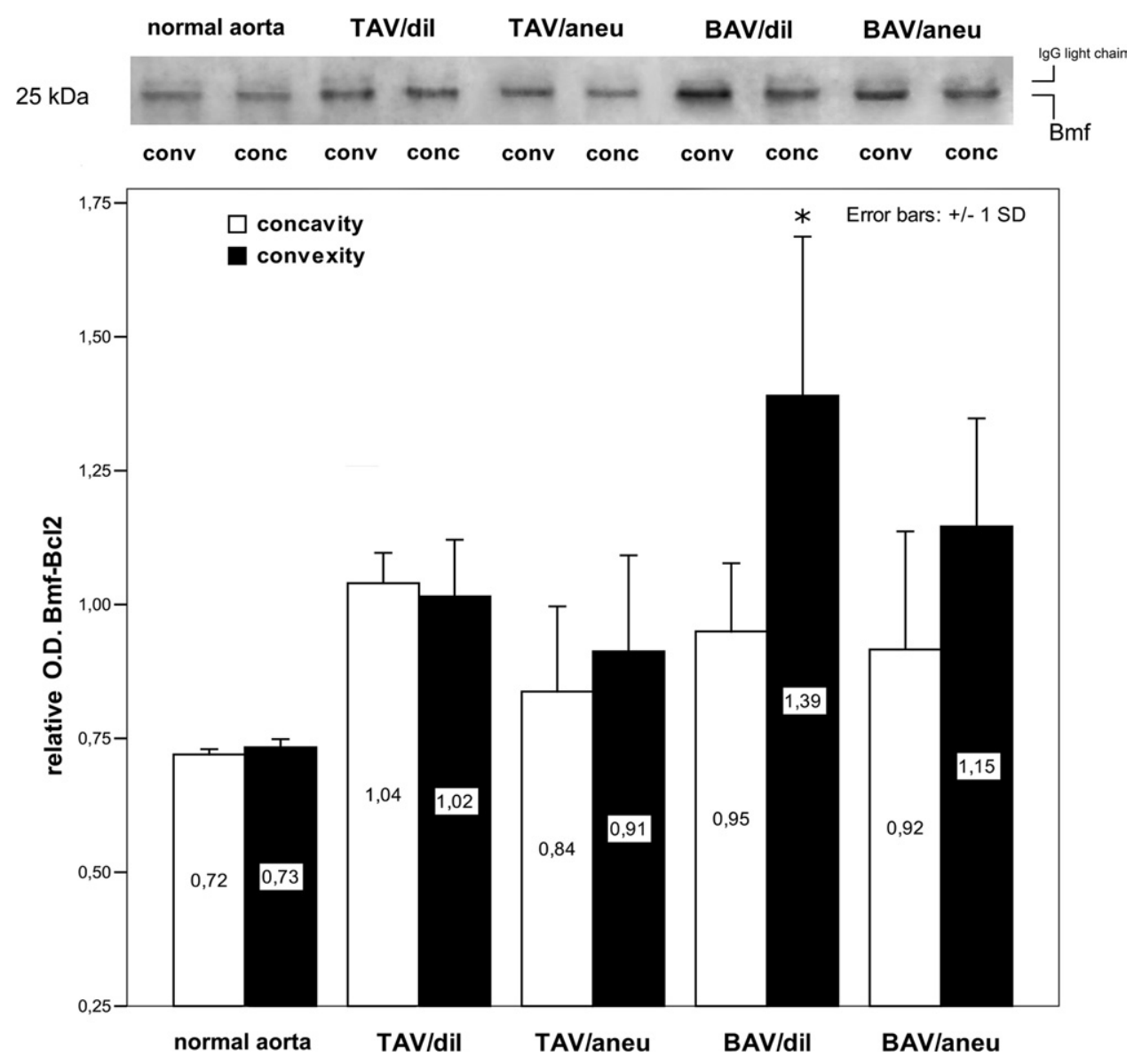

Figure 4. Top, Representative gels showing the results of Bcl-2 immunoprecipitation with Western blot for Bmf; bottom, comparison of $\mathrm{Bmf}-\mathrm{Bcl}-2$ binding levels between subgroups (see text). ${ }^{*} \boldsymbol{P}<.05$ versus normal. Relative $0 . D$., Normalized optical density (arbitrary units); TAV, Tricuspid aortic valve; BAV, bicuspid aortic valve; SD, standard deviation.

aneurysms and nondilated aortas. Kirsch and colleagues ${ }^{18}$ observed higher SMC density in the maximal dilation area of TAV aneurysms than in the transition zone between normal aorta and maximal dilation, thus inferring a temporal pattern of SMC density changes concordant with our observations. However, neither one of those studies ${ }^{17,18}$ investigated SMC apoptosis and proliferation. Concomitant high AI and normal SMC density in our aneurysm specimens could not be explained by concurrent proliferation, since Ki-67 expression was not increased compared with normal. Thus, we suggest that the loss of noncellular components of the media may have quantitatively prevailed on SMC apoptotic loss, for faster progression of ECM breakdown, resulting in apparent normal SMC density, although the absolute cell number must have decreased. Although we could not calculate medial crosssectional areas, ${ }^{17}$ the aortic media was significantly thinned, even with mean diameters smaller than in previous studies. ${ }^{17,18}$ On the other hand, a previous study showed medial cell pro- liferation by $\mathrm{Ki}-67$ staining in aortic dissection, ${ }^{19}$ a condition that may, however, be subtended by different remodeling processes compared with poststenotic dilations. ${ }^{1}$

The differences emerging in the patterns of lesional maturation argue in favor of different mechanisms of early medial remodeling in BAV versus TAV: in BAV aortopathy, apoptosis may be part of the initiating events, whereas in the TAV setting, at least in a proportion of patients, its occurrence is delayed, possibly representing a mechanism, if not a consequence, of dilation itself.

\section{Reciprocal Influences Between ECM and SMC in Aortic Remodeling}

The features of ECM remodeling observed in this study deserve a comment in light of the known paradigms of ECM-SMC interplays. ${ }^{7,8}$ The increase in fibronectin with concomitant decrease in laminin suggests the constitution of a microenvironment that favors SMC phenotype modulation 
from the contractile to the synthetic phenotype. ${ }^{8}$ This suggestion is confirmed by the evidence that the glycoprotein tenascin-C, scarcely detectable in normal aortas, but typically produced by SMCs in synthetic phenotype, ${ }^{4,20}$ was expressed in aneurysms and also in the greater curvature of BAV-related mild dilations. Furthermore, other markers of synthetic phenotype have been found in medial degeneration of the ascending aorta. ${ }^{1}$ The more significant reduction in collagens and laminin in BAV versus TAV dilations is consistent with the greater increase in MMP-2 observed in BAV by Ikonomidis and coworkers. ${ }^{3}$ In our previous study, ${ }^{4}$ collagenolysis in BAV stenotic aorta was suggested by decreased collagen type I, as assessed by Western blot, despite normal expression of collagen type I mRNA. Indeed, tenascin and MMPs are tightly connected in vascular remodeling: tenascin is capable to activate MMPs and, in turn, proteolysed collagen type I stimulates tenascin production. ${ }^{20}$

In the present study, we sought to assess whether ECM changes in BAV aortopathy could exert a causative influence also on SMC apoptosis. Although promoting SMC survival and proliferation is the physiologic function attributed to tenascin in vessel development and response to injury, ${ }^{20}$ it has been shown that in conditions of MMP overexpression, enzymatic degradation of tenascin leads to the exposition of domains in its molecule that are proapoptotic for SMCs. ${ }^{21}$ Similarly, fibronectin is a survival factor for SMCs, ${ }^{7,8}$ although its proteolysis by plasmin and MMPs produces prodetachment/proapoptotic fragments. ${ }^{9}$ Therefore, the MMP repertoire typically expressed in the BAV aorta ${ }^{3}$ may convert a prosurvival ECM into a proapoptotic environment for SMCs. Notably, extrinsic proapoptotic cues are known to be transmitted within the cells through structural rearrangements of the actin cytoskeleton, so that changes in cell-matrix interactions are accompanied by cell shape changes (gross cell rounding, but also subtler disorders) as part of the causative mechanism of apoptosis ("amorphosis"). ${ }^{11,22,23}$ To be able to interact with Bcl-2 and to promote apoptosis, Bmf must be released by its normal site, where it is thought to serve as a sensor of actin cytoskeleton integrity. ${ }^{13,14}$ Our finding of a significant increase in Bmf-Bcl-2 interaction in BAV convexity at an early stage of dilation development strongly suggests that rearrangements in SMC cytoarchitecture may be brought about by early ECM changes and, in turn, convey a proapoptotic signal. ECM-dependent apoptosis does not necessarily require cell detachment (anoikis proper): alterations of matrix geometrical organization or physical properties can trigger this mechanism of programmed cell death even without detachment. ${ }^{9-11,13}$ Indeed, we observed a reduction of Bcl-2 mRNA, whose expression is regulated also by matrix-dependent cytoskeletal changes. ${ }^{22,23}$ The fact that Bmf-Bcl-2 did not further increase in larger aneurysms, despite the increment in AI, suggests that late apoptosis, both in TAV aneurysms and in BAV concavity, may result from other pathways. Our study did not explore, for example, the apoptotic pathway of cytokine signaling, and the possible involvement of inflammatory mediators ${ }^{17,18}$ should be investigated in early versus late lesions. However, late apoptosis may be not a causative factor but rather a consequence of aneurysm growth itself, and another possible cause could be increased wall strain owing to enlargement and loss of elasticity. ${ }^{17}$ Apoptosis in response to cyclic strain has been shown to occur in well-differentiated contractile SMCs, but not in those that have undergone phenotype change ${ }^{24}$ : consistently, we found no AI elevation in BAV aneurysm convexity, where a greater expression of ECM proteins promoting phenotype switch was observed.

\section{A Pathogenetic Hypothesis}

The asymmetrical spatial pattern of ECM and SMC changes uniquely found in early BAV dilations introduces, in the long-standing debate on the pathogenesis of BAV-related aortic disease, evidence in favor of a determinant or at least contributory role of hemodynamics. A BAV stenosis, being asymmetric in geometry, produces an eccentric jet yielding more severe flow alterations in the ascending aorta than TAV stenosis with similar gradients and valve areas. ${ }^{25}$ BAV-related turbulences have been shown to produce uneven wall stress distribution, with overload at the aortic right anterolateral wall (convexity). ${ }^{26}$ Conversely, a hypothetical inborn wall defect $^{2}$ sufficient to cause dilation should produce a remodeling process uniformly involving the whole aortic circumference, which was denied by the present study. Thus, we hypothesize that altered flow (even though not necessarily a "clinically" altered flow ${ }^{25,26}$ ) could be either the triggering cause of early wall lesions or the factor required for local expression of a latent defect. Both in vitro and in vivo studies have shown SMC phenotype change, ${ }^{27}$ fibronectin and tenascin production, ${ }^{27}$ and increased MMP expression and activity ${ }^{28}$ to be elicited by undue biomechanical stress, as well as apoptotic SMC death, with reduced $\mathrm{Bcl}-2$ expression, ${ }^{24}$ as found in the present study, and increased p53 expression, ${ }^{29}$ as found by Ihling and colleagues ${ }^{19}$ in medial degeneration. In our hypothesis, stress-induced early ECM changes unique to BAV convexity promote early SMC apoptosis, at least in part through Bmf-Bcl-2 interaction, leading to progressive, typically asymmetric aortic enlargement.

However, further work is needed to verify this theory. The possibility cannot be discarded that BAV might be associated with a congenital anomaly of some component of matrix signaling pathways, and flow disturbances at the convexity could merely accelerate locally the degenerative process, however destined to involve also the concavity within a longer time span. 


\section{Study Limitations}

A first limitation of the present study concerns the reference control group ("normal aorta"): the most appropriate control subjects for comparison with patients with BAV aortopathy should have been the nonstenotic BAV with normal-sized aorta, a setting that was not represented in our surgical specimens. Second, the reason for the choice of Bmf-Bcl-2 measurement as the sign of proapoptotic cytoskeleton rearrangement was that $\mathrm{Bmf}$ release does not occur in response to other types of apoptotic stimuli in vivo. ${ }^{10,13}$ Cell-matrix detachment has never been demonstrated in medial degeneration; thus, our starting hypothesis was that altered matrix solidity could be rather responsible for proapoptotic signaling. ${ }^{11,13}$ However, whether cell anchorage is lost or not remains to be discerned, and to this scope, caspase 8 and integrins may represent interesting targets: caspase 8 activity increases after loss of integrin-mediated anchorage, but Bmf can be released without caspase 8 activation in the case of cytoskeleton rearrangement without detachment. ${ }^{14,22}$ Finally, smooth muscle $\alpha$-actin and other SMC markers can be expressed also by myofibroblasts, migrating from the adventitial layer to the media during vascular remodeling, for example, in response to mechanical injury. ${ }^{30}$ Thus, our study, as well as the previous ones using smooth muscle actin staining, ${ }^{1,2,17-19}$ could not differentiate myofibroblasts from SMCs in the media, and the assessment of the role of resident cell types other than SMCs awaits further study.

\section{Conclusions}

Spatiotemporal patterns of ECM and SMC changes in the dilating ascending aorta were found to differ between BAV and TAV stenosis, because matrix disruption and SMC apoptosis were earlier alterations in BAV than those in $\mathrm{TAV}$, preceding overt enlargement of the vessel and peculiarly located in the aortic wall area of the greater curvature, or convexity. TAV-related dilation constitutes a more heterogeneous phenomenon, likely resulting from a variety of causative mechanisms. The processes of cell-matrix signaling represent a potential field of investigation for future research aiming to elucidate the etiology of BAV-related ascending aorta dilation.

\section{References}

1. Lesauskaite V, Tanganelli P, Sassi C, Neri E, Diciolla F, Ivanoviene L, et al. Smooth muscle cells of the media in the dilatative pathology of ascending thoracic aorta: morphology, immunoreactivity for osteopontin, matrix metalloproteinases, and their inhibitors. Hum Pathol. 2001; $32: 1003-11$

2. Bonderman D, Gharehbaghi-Schnell E, Wollenek G, Maurer G, Baumgartner H, Lang IM. Mechanisms underlying aortic dilatation in congenital aortic valve malformation. Circulation. 1999;99:2138-43.

3. Ikonomidis JS, Jones JA, Barbour JR, Stroud RE, Clark LL, Kaplan BS, et al. Expression of matrix metalloproteinases and endogenous inhibitors within ascending aortic aneurysms of patients with bicuspid or tricuspid aortic valves. J Thorac Cardiovasc Surg. 2007;133: 1028-36.

4. Cotrufo M, Della Corte A, De Santo LS, Quarto C, De Feo M, Romano $\mathrm{G}$, et al. Different patterns of extracellular matrix protein expression in the convexity and the concavity of the dilated aorta with bicuspid aortic valve: preliminary results. J Thorac Cardiovasc Surg. 2005;130: 504-11.

5. Della Corte A, Bancone C, Quarto C, Dialetto G, Covino FE, Scardone $\mathrm{M}$, et al. Predictors of ascending aortic dilatation with bicuspid aortic valve: a wide spectrum of disease expression. Eur J Cardiothorac Surg. 2007;31:397-405.

6. Davies RR, Kaple RK, Mandapati D, Gallo A, Botta DM Jr, Elefteriades JA, et al. Natural history of ascending aortic aneurysms in the setting of an unreplaced bicuspid aortic valve. Ann Thorac Surg. 2007;83:1338-44.

7. Zhang Z, Vuori K, Reed JC, Ruoslahti E. The alpha 5 beta 1 integrin supports survival of cells on fibronectin and up-regulates Bcl-2 expression. Proc Natl Acad Sci U S A. 1995;92:6161-5.

8. Thyberg J, Hultgardh-Nilsson A. Fibronectin and the basement membrane components laminin and collagen type IV influence the phenotypic properties of subcultured rat aortic smooth muscle cells differently. Cell Tissue Res. 1994;276:263-71.

9. Michel JB. Anoikis in the cardiovascular system: known and unknown extracellular mediators. Arterioscler Thromb Vasc Biol. 2003;23: 2146-54.

10. Martin SS, Vuori K. Regulation of Bcl-2 proteins during anoikis and amorphosis. Biochim Biophys Acta. 2004;1692:145-57.

11. Chen CS, Mrksich M, Huang S, Whitesides GM, Ingber DE. Geometric control of cell life and death. Science. 1997;276:1425-8.

12. Scholzen T, Gerdes J. The Ki-67 protein: from the known and the unknown. J Cell Physiol. 2000;182:311-22.

13. Puthalakath H, Villunger A, O'Reilly LA, Beaumont JG, Coultas L, Cheney RE, et al. Bmf: a proapoptotic $\mathrm{BH} 3$-only protein regulated by interaction with the myosin $\mathrm{V}$ actin motor complex, activated by anoikis. Science. 2001;293:1829-32.

14. Frisch SM, Screaton RA. Anoikis mechanisms. Curr Opin Cell Biol. 2001;13:555-62.

15. Borger MA, Preston M, Ivanov J, Fedak PW, Davierwala P, Armstrong $\mathrm{S}$, et al. Should the ascending aorta be replaced more frequently in patients with bicuspid aortic valve disease? J Thorac Cardiovasc Surg. 2004;128:677-83.

16. McKellar SH, Tester DJ, Yagubyan M, Majumdar R, Ackerman MJ, Sundt TM. Novel NOTCH1 mutations in patients with bicuspid aortic valve disease and thoracic aortic aneurysms. J Thorac Cardiovasc Surg. 2007;134:290-6.

17. Tang PC, Coady MA, Lovoulos C, Dardik A, Aslan M, Elefteriades JA, et al. Hyperplastic cellular remodeling of the media in ascending thoracic aortic aneurysms. Circulation. 2005;112:1098-105.

18. Kirsch EW, Radu NC, Gervais M, Allaire E, Loisance DY. Heterogeneity in the remodeling of aneurysms of the ascending aorta with tricuspid aortic valves. J Thorac Cardiovasc Surg. 2006;132:1010-6.

19. Ihling C, Szombathy T, Nampoothiri K, Haendeler J, Beyersdorf F, Uhl M, et al. Cystic medial degeneration of the aorta is associated with p53 accumulation, Bax upregulation, apoptotic cell death, and cell proliferation. Heart. 1999;82:286-93.

20. Jones PL, Jones FS, Zhou B, Rabinovitch M. Induction of vascular smooth muscle cell tenascin-C gene expression by denatured type I collagen is dependent upon a beta3 integrin-mediated mitogen-activated protein kinase pathway and a 122-base pair promoter element. J Cell Sci. 1999;112:435-45.

21. Wallner K, Li C, Shah PK, Wu KJ, Schwartz SM, Sharifi BG. EGF-like domain of tenascin-C is proapoptotic for cultured smooth muscle cells. Arterioscler Thromb Vasc Biol. 2004;24:1416-21.

22. Flusberg DA, Numaguchi Y, Ingber DE. Cooperative control of Akt phosphorylation, bcl-2 expression, and apoptosis by cytoskeletal microfilaments and microtubules in capillary endothelial cells. Mol Biol Cell. 2001;12:3087-94.

23. Boudreau NJ, Jones PL. Extracellular matrix and integrin signalling: the shape of things to come. Biochem J. 1999;339:481-8.

24. Birney YA, Sweeney CH, Cappadona CR, Sitzmann JV, Cummins PM, Redmond EM, et al. Pulse pressure-induced transmural fluid flux 
increases bovine aortic smooth muscle cell apoptosis in a mitogen activated protein kinase dependent manner. J Vasc Res. 2004; 41:364-74.

25. Donal E, Novaro GM, Deserranno D, Popovic ZB, Greenberg NL, Richards KE, et al. Planimetric assessment of anatomic valve area overestimates effective orifice area in bicuspid aortic stenosis. Am J Soc Echocardiogr. 2005; 18:1392-8.

26. Robicsek F, Thubrikar MJ, Cook JW, Fowler B. The congenitally bicuspid aortic valve: How does it function? Why does it fail? Ann Thorac Surg. 2004;77:177-85.

27. Williams B. Mechanical influences on vascular smooth muscle cell function. J Hypertens. 1998;16:1921-9.

28. Grote K, Flach I, Luchtefeld M, Akin E, Holland SM, Drexler H, et al. Mechanical stretch enhances mRNA expression and proenzyme release of matrix metalloproteinase-2 (MMP-2) via $\mathrm{NAD}(\mathrm{P}) \mathrm{H}$ oxidasederived reactive oxygen species. Circ Res. 2003;92:80-6.

29. Wernig F, Mayr M, Xu Q. Mechanical stretch-induced apoptosis in smooth muscle cells is mediated by $\beta$-1-integrin signalling pathways. Hypertension. 2003;41;903-11.

30. Chambers RC, Leoni P, Kaminski N, Laurent GJ, Heller RA. Global expression profiling of fibroblast responses to transforming growth factor-beta1 reveals the induction of inhibitor of differentiation- 1 and provides evidence of smooth muscle cell phenotypic switching. Am J Pathol. 2003;162:533-46.

\section{Discussion}

John S. Ikonomidis (Charleston, SC). I congratulate Dr Della Corte for an outstanding study, which showed temporospatial changes in extracellular proteins, vascular smooth muscle density, and indexes of apoptosis in normal or aneurysmal ascending aortic specimens taken from patients with normal or stenotic BAVs or TAVs.

In particular, and in keeping with previous studies from this group, extracellular matrix protein changes were particularly important in the convexity of aneurysmal ascending aorta associated with BAVs. In addition, evidence of early SMC apoptosis was demonstrated. Of particular relevance here is evidence presented that anoikis, or apoptosis, which is induced by the loss of integrinmediated cellular attachments, participates in the process of SMC attrition as the aneurysm develops. This, of course, makes sense because extracellular matrix remodeling and its attendant changes of cellular matrix interactions are universal features of aortic aneurysm development.

I have 4 questions: Your extracellular protein assessments were conducted by immunohistochemical techniques. Elastin was not included in these results but is known to cause significant autofluorescence in these studies. How did you correct for this phenomenon when you were assessing the other proteins?

Dr Della Corte. Thank you, Dr Ikonomidis. My coworkers from the biomorphology department used to pre-tract tissues with toluidine blue solution and then they used a very selective green filter for fluorescence microscopy to obviate elastin autofluorescence.

We have already published our preliminary results with ECM protein assessment using both immunohistochemistry and Western blot, which is not affected by elastin autofluorescence, and the results of the two methods were highly consistent.

Dr Ikonomidis. Second question: Your data suggest that SMC apoptosis is primed and occurs early in the course of aneurysm development. However, in the later phase of aneurysm development, you demonstrate an increase in SMC density. How do you explain these findings and did you examine fibroblast profiles as a second resident cell within the aortic aneurysm wall?

Dr Della Corte. We didn't look at other resident cell types in this study, and this may be an interesting point for future research. However, both the evidence of smooth muscle cell loss in an early phase of dilation with bicuspid valves and that of an increase in smooth muscle cell density in aneurysms compared to small dilations (resulting in near-normal cell density) have been already reported in two different studies by other authors. Here we have tried to suggest a common interpretation for those two findings in the light of cell-matrix interactions. Cell-matrix signaling may be responsible for early apoptosis with the anoikis mechanism, as we have demonstrated it, but also, in the later phase of aneurysm development, the effect of increased morphogenetic pro-survival proteins, like fibronectin and tenascin, may explain smooth muscle cell density increase.

Dr Ikonomidis. Were you able to stratify these data relative to the degree of aortic stenosis?

Dr Della Corte. We tried to compare subgroups of patients with different degrees of valve stenosis, but no difference in aortic wall changes emerged. However, we expected such results, because other factors may be important besides the degree of aortic valve stenosis, as for example the time duration of valve disease.

Dr Ikonomidis. Last, have you related any of your changes to profiles of known protease systems such as MMPs?

Dr Della Corte. We observed a greater decrease in collagen in BAV compared with TAV patients, and this may be consistent with the increase in MMP-2 expression that is quite unique to the TAV setting, or at least greater in BAV than in TAV patients.

Similarly, our finding of increased tenascin and fibronectin expression may be consistent with the decrease in MMP-3 that your group has demonstrated in the setting of bicuspid aortopathy. However, we should keep in mind that protein amount is the result of a fine balance between production and degradation of proteins. 


\section{Appendix E1}

Polymerase chain reactions (PCRs) were performed with the following program:

1. Initial denaturation for 5 minutes at $95^{\circ} \mathrm{C}$

2. Denaturation for 45 seconds at $95^{\circ} \mathrm{C}$

3. Annealing for 1 minute at $59^{\circ} \mathrm{C}$

4. Extension for 2 minutes at $68^{\circ} \mathrm{C}$

5. Final extension at $68^{\circ} \mathrm{C}$ for 5 minutes

The following primers of the $\mathrm{Bcl}-2$ gene and the glyceraldehyde-3-phosphate dehydrogenase (GAPDH) housekeeping gene were used for PCR (expected size of the amplification product):
- Bcl-2: 5' -ATGCCAAGGGGGAAACACCAG-3' (forward primer)

- 5'-AGACAGCCAGGAGAAATCAAA-3' (reverse primer) (831 bp)

- GAPDH: 5'-CACCATCTTCCAGGAGCGAG-3' (forward primer)

- 5' -TCACGCCACAGTTTCCCGGA-3' (reverse primer) (372 bp) 
TABLE E1. Within groups of aortic dimensions (small-size dilations and aneurysms), BAV and TAV patients were homogeneous in terms of age, prevalence of hypertension, aortic valve stenosis parameters, and aortic dimensions

\begin{tabular}{|c|c|c|c|c|c|c|}
\hline & TAV/dilation $(n=7)$ & BAV/dilation $(n=7)$ & $P^{*}$ & TAV/aneurysm $(n=5)$ & BAV/aneurysm $(n=7)$ & $P+$ \\
\hline Age (y) & $60 \pm 9$ & $54 \pm 14$ & .37 & $67 \pm 11$ & $60 \pm 15$ & .47 \\
\hline Hypertension (rate) & $57 \%$ & $43 \%$ & .50 & $60 \%$ & $57 \%$ & .69 \\
\hline Mean gradient $(\mathrm{mm} \mathrm{Hg})$ & $76 \pm 32$ & $63 \pm 12$ & .47 & $40 \pm 5$ & $47 \pm 9$ & .11 \\
\hline EOAI $\left(\mathrm{cm}^{2} / \mathrm{m}^{2}\right)$ & $0.31 \pm 0.02$ & $0.35 \pm 0.1$ & .58 & $0.42 \pm 0.03$ & $0.37 \pm 0.07$ & .39 \\
\hline Mild aortic regurgitation (rate) & $29 \%$ & $43 \%$ & .50 & $60 \%$ & $57 \%$ & .69 \\
\hline Sinuses $(\mathrm{cm})$ & $3.2 \pm 0.4$ & $3.5 \pm 0.4$ & .21 & $3.9 \pm 0.6$ & $3.8 \pm 0.7$ & .91 \\
\hline Aortic ratio & $1.19 \pm 0.15$ & $1.15 \pm 0.17$ & .68 & $1.54 \pm 0.14$ & $1.55 \pm 0.12$ & .92 \\
\hline
\end{tabular}

TAV, Tricuspid aortic valve; $B A V$, bicuspid aortic valve; EOAl, effective orifice area index. ${ }^{*} \mathrm{TAV} / \mathrm{dilation}$ versus BAV/dilation; †TAV/aneurysm versus BAV/aneurysm. Additionally, no significant difference $(P>.05)$ was found when comparing BAV dilations versus aneurysms and TAV dilations versus aneurysms in terms of age and stenosis severity.

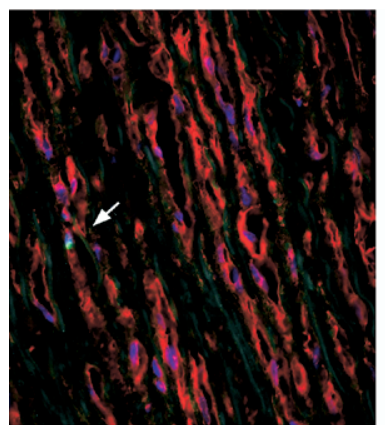

normal aorta

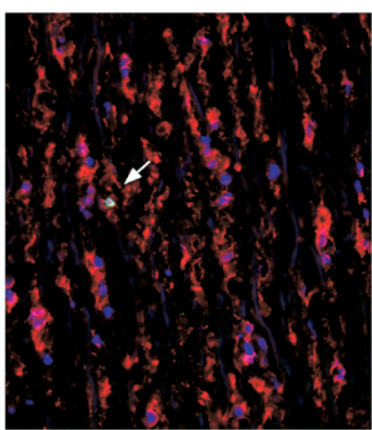

BAV aneurysm

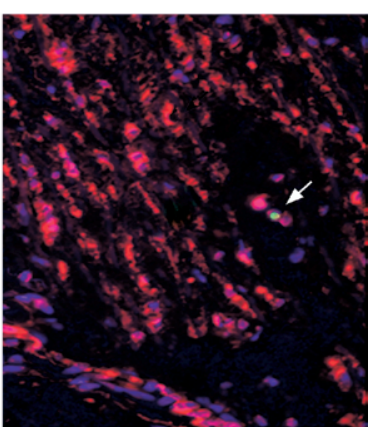

TAV aneurysm

Figure E1. Representative images of Ki-67 staining (arrows) showing no difference in SMC proliferative index between a normal aorta, a BAV aneurysm, and a TAV aneurysm. TAV, Tricuspid aortic valve; BAV, bicuspid aortic valve. 